\title{
MiR-1203 is involved in hepatocellular carcinoma metastases and indicates a poor prognosis
}

\author{
J. SHI ${ }^{1,2, *}, \mathrm{X} . \mathrm{LI}^{3, \sharp}, \mathrm{Y} . \mathrm{HU}^{4, *}, \mathrm{~F} . \mathrm{ZHANG}{ }^{2}, \mathrm{X} . \mathrm{LV}^{2}$, X. ZHANG ${ }^{2}$, Q. CHEN ${ }^{2, *}, \mathrm{~S} . \mathrm{HU}^{1, *}$ \\ ${ }^{1}$ Department of General Surgery, Shandong Provincial Qianfoshan Hospital, Shandong University, Jinan, Shandong 250014, China; ${ }^{2}$ Depart- \\ ment of Hepatobiliary Surgery, Binzhou Medical University Hospital, Binzhou 256603, China; ${ }^{3}$ Department of Pediatrics, Binzhou Medical \\ University Hospital, Binzhou 256603, China; ${ }^{4}$ Department of Hepatobiliary and Pancreatic Surgery, Changzhou Second People's Hospital Affili- \\ ated to Nanjing Medical University, Changzhou 213000, China
}

*Correspondence: hu_sanyuan@sina.com; dr_cqp@163.com

${ }^{*}$ Contributed equally to this work.

Received April 14, 2019 / Accepted June 26, 2019

\begin{abstract}
Non-coding RNAs, especially miRNAs, have been shown to be important regulators in multiple human diseases, including malignant tumors, congenital disease, and autoimmune disease. In this study, we screened the metastasis-associated miRNAs in hepatocellular carcinoma (HCC). Based on the miRNA microarray screening, miR-1203 was confirmed to be the most significant miRNA and was also highly associated with HCC metastases. Bioinformatics prediction indicated direct binding of miR-1203 in SOCS3, which was also confirmed by a dual luciferase reporter assay, resulting in suppression of SOCS3. Increased miR-1203 also promoted invasion of HCC cells through suppressing SOCS3, while no effect on cell proliferation or apoptosis was detected. Circulating expression of miR-1203 and SOCS3 might serve as a predictor of metastases and poor prognosis in HCC patients. In conclusion, miR-1203 might promote HCC metastasis by decreasing SOCS3. MiR-1203 predicts a poor outcome in HCC patients and thus might serve as a potential therapeutic target for the prevention of HCC.
\end{abstract}

Key words: miRNA, metastasis, biomarker, HCC, SOCS3

Hepatocellular carcinoma (HCC) is the fifth most common cancer worldwide, and the incidence of HCC has been rapidly increasing in the last decade [1-3]. HCC is characterized by aggressive malignancy, invasiveness (especially towards intrahepatic invasion), and frequent recurrence after surgical resection $[4,5]$. Metastasis is a multistep process and identifying the mechanisms responsible for cancer metastasis could facilitate the development of novel therapeutic strategies. Accumulating evidence indicates that epithelial-mesenchymal transition (EMT) is the first step toward metastasis [6]. During the initiation and progression of hepatocarcinogenesis, multiple genetic and epigenetic events accumulate, leading to deregulated expression of various cellular genes $[7,8]$. Therefore, identifying biomarkers, especially for predicting the metastasis of HCC, is critical for early detection and prognosis, which can remarkably improve survival rates.

Several studies have revealed that miRNAs may serve as fingerprints for multiple human malignant tumors, including gastric cancer, colon cancer, lung cancer, and hepatocellular carcinoma [9-12]. In some cases, miRNAs can also predict poor prognosis $[13,14]$. MiRNAs are expressed in a tissue- specific manner and play essential roles in the regulation of oncogenes and tumor suppressor genes [15]. MiRNAs induce mRNA degradation or suppress protein translation by directly binding to the 3' untranslated regions (UTRs) of target mRNAs [16]. For example, miR-338-3p has been shown to suppress EMT and metastases via inhibition of the SHH/Gli1 pathway and direct binding of N-cadherin [17].

Suppressor of cytokine signaling 3 (SOCS3) is a target gene for STAT3 and also a member of the STAT-induced STAT inhibitor family, which functions as a cytokine-inducible negative regulator of cytokine signaling [18]. The expression of SOCS3 is induced by various cytokines, including IL-6. SOCS3 can bind to JAK2 kinase and inhibit its activity, thus blocking the activation of STAT3 and controlling the IL-6/STAT3 signaling pathway by a classic negative feedback loop [19]. Accumulating evidence has revealed that SOCS3 is epigenetically silenced, resulting in the hyper-activation of STAT3 in many cancers, such as cholangiocarcinoma, head and neck squamous cell carcinoma and HCC [20]. Loss of SOCS3 in HCC is associated with STAT3 over-phosphorylation and poor prognosis. Accumulating studies support that 
inhibition of SOCS3 expression promoted STAT3 activation, enhanced hepatic fibrosis, increased proliferation and tumor aggressiveness [21].

In this study we performed high-throughput screening of potential miRNAs involved in HCC metastases using tissues samples from HCC patients with or without metastases. A multiple analysis and gain-and-loss function assays were used to confirm dysregulated miRNAs and potential functions.

\section{Patients and methods}

Patient samples. Study data were obtained from 80 patients who presented between March 2013 and October 2018 at Binzhou Medical University Hospital. The research protocol was approved by the Shandong University and Binzhou Medical University. Written informed consent was obtained from every participant. All patients were diagnosed with HCC based on pathologic examination. Thirty-four patients were confirmed to have no metastases based on pathologic examination, while 46 patients had intrahepatic metastases or portal vein tumor thrombi. We also evaluated 8 non-tumorous liver tissues, which were obtained from patients with hemangiomas and 7 tissues from patients with liver cirrhosis, who served as the control group. Informed consent for use of blood and tissues was obtained prior to surgery. The clinicopathologic characteristics included age, gender, ALT and AFP levels, tumor size, vascular invasion, and histological grade. The clinical samples used in this study included peripheral blood obtained from patients before surgery or chemotherapy. After we obtained the blood, the serum was stored, while the white blood cells were used for RNA extraction.

Microarray assay detection and bioinformatics analyses. Twenty samples were used for screening, including 3 liver tissue samples from patients with hemangiomas (normal controls), 4 samples from patients with cirrhosis (negative controls), 4 samples from patients with HCC without metastases, 4 samples from patients with HCC and portal vein tumor thrombi (PVTT), and 5 samples from patients with HCC with intrahepatic metastases (IM). The tissues obtained from the patients described above were used to extract the total RNA from three samples in each group and amplified. In the screening stage, TLDA Chips (Life Technologies, CA, USA) were used to screen differentially expressed miRNAs. Megaplex RT and pre-amplification reactions were run according to the manufacturer's protocol. TE was added to the PreAmp product and $9 \mu \mathrm{l}$ of diluted PreAmp product was used to run the RT-PCR reactions by dispensing $100 \mu$ lof the PCR reaction mix into each port of the TaqMan MicroRNA Array. The default PCR procedure was used and the analysis was performed using RQ manager software (Life Technologies). Bioinformatics analyses were conducted using a VENNY system.

Bioinformatics analysis. Bioinformatics analysis was performed using miRNA-associated target gene predic- tion databases, including Targetscan (www.targetscan.org/), PicTar (pictar.mdc-berlin.de/), and miRTarget (mirdb.org/). The mature sequence of miR-1203 was used as the input. The candidate target genes were screened according to the rank score of the databases.

Quantitative real time polymerase chain reaction (qRT-PCR). MiRNA-specific TaqMan MicroRNA Assays (Life Technologies) were used for the miRNAs. The data obtained were calculated using the $\Delta \mathrm{Cq}$ method (CT value of the target gene minus the CT value of GAPDH), as described. The relative expression corresponded to the $2^{\Delta \mathrm{Cq}}$.

Cell invasion assay. For the Transwell assay, the chamber was treated with Matrigel before cell inoculation. A $100 \mu \mathrm{l}$ cell suspension in serum-free medium was seeded to the upper chamber and the cells were stained with crystal violet staining solution (Beyotime, Nantong, China). Migrated cells were counted using Image-pro Plus 6.0, while the number of cells in the normal control group were normalized to 1 .

Western blot. Proteins were extracted from tissues or cultured cells using RIPA buffer containing phenylmethanesulfonylfluoride (PMSF; Beyotime). An equal amount of protein $(100 \mu \mathrm{g})$ was separated with $7.5 \% / 12.5 \%$ sodium dodecyl sulphate polyacrylamide gel electrophoresis (SDS-PAGE) and transferred to polyvinylidene fluoride (PVDF) membranes. The blots were developed using ECL reagent (Millipore, MA, USA). An equal amount of protein loading in each lane was confirmed using GAPDH antibody (Abcam, Cambridge, UK).

Luciferase reporter gene assay. The 3'-UTR sequence of SOCS3 (either wild or mutant type) was inserted into the pGL3 promoter vector. For the reporter assay, HepG2 and Hep3B cells were plated onto 24-well plates. A Renilla luciferase vector (pRL-SV40, 5ng) was co-transfected to normalize the differences in transfection efficiency.

Circulating miRNA detection. The miRNA specific TaqMan MicroRNA Assay (Life Technologies) was used for plasma miRNAs. The probe was purchased from Applied Biosystems (Life Technologies). One microgram of the total RNA was reverse transcribed with a TaqMan MicroRNA Reverse Transcription Kit (Life Technologies). RT-PCR was performed using the ABI 7900 HT Real-Time PCR System (Life Technologies). The reactions were initiated in a 384-well optical plate at $95^{\circ} \mathrm{C}$ for $5 \mathrm{~min}$, followed by 40 cycles at $95^{\circ} \mathrm{C}$ for $15 \mathrm{~s}$ and $60^{\circ} \mathrm{C}$ for $1 \mathrm{~min}$. The levels of miRNA expression were normalized to miR-16 as an internal control in agreement with other publications [8].

Statistical analysis. Data are presented as the mean (SEM). A $\chi^{2}$ test, Student's t-test, and analysis of variance (ANOVA) were used to evaluate statistical differences in demographic and clinical characteristics. The experimental assays were repeated three times in triplicate. We also tested the homoscedasticity in all the data obtained. After confirming homoscedasticity, parametric tests ( $\mathrm{t}$-test and ANOVA) were applied. Kaplan-Meier survival curves were plotted, and a log rank test was performed. The significance 
of various variables for survival was analyzed using a Cox proportional hazards model in a multivariate analysis. Statistical analysis was performed using STATA 9.2, and presented with Graph PAD prism software. In all cases, $\mathrm{p}<0.05$ was considered statistically significant.

\section{Results}

Upregulated miR-1203 is highly associated with HCC metastasis. Because the miRNA has been shown to be highly associated with HCC metastases, we applied microarraybased screening. As presented in Figure 1A, 20 samples obtained from patients were used for screening, including 3 liver samples from hemangioma patients (normal control), 4 samples from patients with cirrhosis (negative control), 4 samples from HCC patients without metastases, 4 samples with HCC and PVTT, and 5 samples from HCC patients with IM. An abnormal profile of miRNA expression is presented in Figure 1A. We next compared the HCC patients with and without metastases (with PVTT and IM) with the control or cirrhosis groups. Furthermore, Venny toolbox was also used, as shown in Figure 1B. We found 8 dysregulated miRNAs in samples from patients with metastases compared to patients without metastases. We further detected the expression of 8 potential miRNAs in a larger cohort, among which we found that miR-1203 had the most significant upregulation in HCC tissues from HCC patients with PVTT and IM (Figure 1C).

Clinical information was collected to detect whether the aberrant expression of miR-1203 associated with clinicopathologic characteristics. The median value was set as the cut-off to classify the level of miR-1203 expression. The tumor size was grouped using $3 \mathrm{~cm}$ as the cut-off, which was the threshold indicating small HCCs. As listed in Table 1, we found that the expression of $\mathrm{miR}-1203$ was highly correlated with vascular invasion $(\mathrm{p}=0.007)$.

miR-1203 suppressed SOCS3 via direct binding. To further study the role of miR-1203 in HCC, bioinformatics analysis was conducted to predict the putative target genes of
A

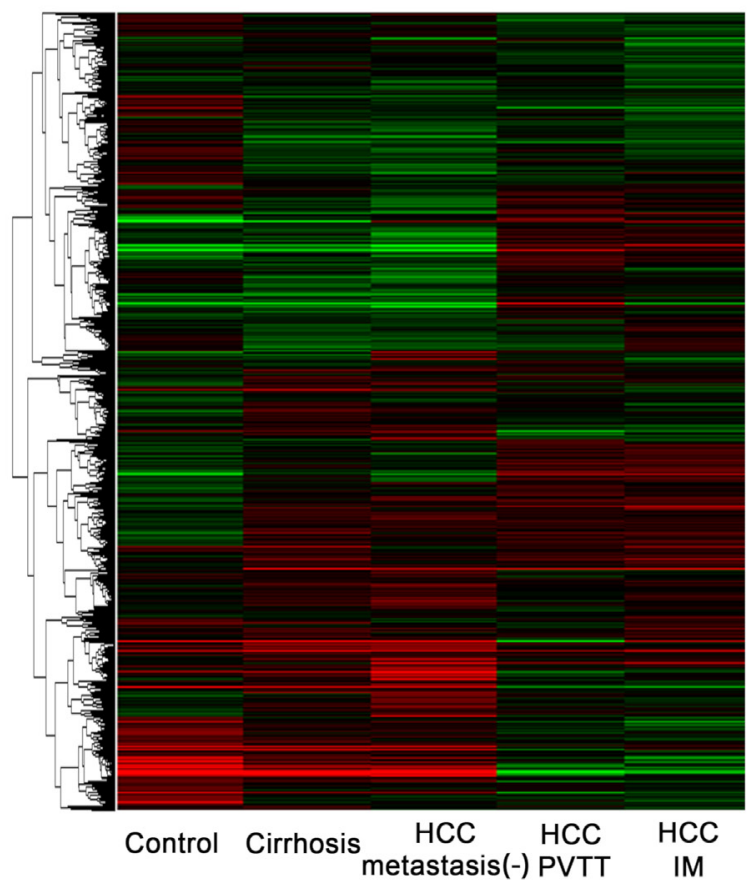

B

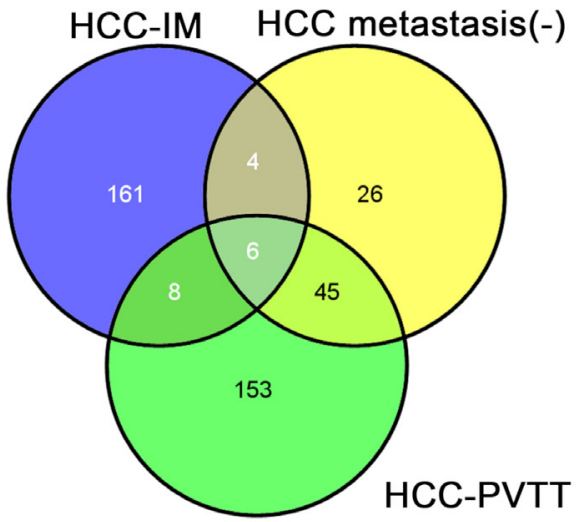

C

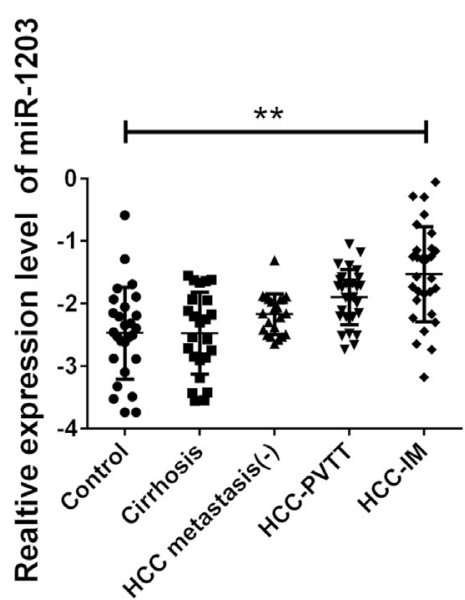

Figure 1. The clinicopathological analysis of miR-1203 expression in HCC patients. A) Cluster analysis of aberrant expressed miRNAs in tissue samples. PVTT indicated the HCC with portal vein tumor thrombus, while IM indicated intrahepatic metastasis. The heatmap was constructed by using the expression level of miRNAs in different groups. The Cluster 3.0 and Treeview were applied. The miR-1203 was labelled with red. B) Venny diagram in three groups. Each was compared with both the control group and cirrhosis group. C) Relative expression of miR-1203 in patients (n=80). Data are presented as mean \pm SEM. ${ }^{\star}$ indicates $\mathrm{p}<0.05$. 
Table 1. Correlation between miR-1203 expression and clinicopathological characteristics of HCC patients $(n=80)$.

\begin{tabular}{|c|c|c|c|}
\hline Characteristics & $\begin{array}{l}\text { Low Expression }{ }^{\mathrm{a}} \\
\mathrm{n}=40\end{array}$ & $\begin{array}{c}\text { High Expression }^{\mathrm{a}} \\
\mathrm{n}=40\end{array}$ & p-value \\
\hline \multicolumn{4}{|l|}{ Age (years) } \\
\hline$<60$ & 20 & 19 & 0.823 \\
\hline$\geq 60$ & 20 & 21 & \\
\hline \multicolumn{4}{|l|}{ Gender } \\
\hline Male & 29 & 23 & 0.160 \\
\hline Female & 11 & 17 & \\
\hline \multicolumn{4}{|l|}{$\operatorname{ALT}(\mathbf{U} / \mathbf{L})$} \\
\hline$\leq 45$ & 11 & 13 & 0.626 \\
\hline$>45$ & 29 & 27 & \\
\hline \multicolumn{4}{|l|}{$\operatorname{AFP}(\mathbf{n g} / \mathrm{ml})$} \\
\hline$\leq 13.6$ & 22 & 21 & 0.823 \\
\hline$>13.6$ & 18 & 19 & \\
\hline \multicolumn{4}{|l|}{ Tumor size $(\mathrm{cm})$} \\
\hline$\leq 3$ & 1 & 3 & 0.305 \\
\hline$>3$ & 39 & 37 & \\
\hline \multicolumn{4}{|c|}{ Vascular invasion } \\
\hline Absent & 27 & 15 & 0.007 \\
\hline Present & 13 & 25 & \\
\hline
\end{tabular}

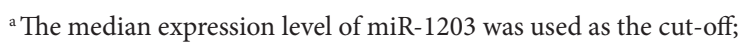

${ }^{*}$ indicates p-value $<0.05$
miR-1203. The prediction results were shared in TargetScan, PicTar, and miRTarget software, and the candidate target genes for miR-1203 are listed in Table 2. The expression of candidate gene was detected after treatment with either miR-1203 mimics or inhibitor. As presented in Figure 2, only SOCS3 was suppressed by miR-1203 and increased by the loss of miR-1203, thus, we chose SOCS3 as the target gene. The expression correction between miR-1203 and SOCS3 was further analyzed, as presented in Figure 3A, and a reversed correction was obtained. To examine whether miR-1203 regulates SOCS3 via 3'UTR binding, the expression of miR-1203 and SOCS3 was firstly confirmed in HCC cell lines, as presented in Figure 3B and C. We next synthesized the full length of the 3'UTR region of SOCS3, which was then inserted into a pGL3 plasmid. The mutant type at the predicted binding site was also cloned (Figure 3D). We thus chose HepG2 and Hep3B as models for further transfection. The two cell lines were transfected with each plasmid, along with miR-1203 mimics, and luciferase activity was measured $48 \mathrm{~h}$ post-transfection. The data suggest that the miR-1203 target sites in SOCS3 were responsible for the decreased luciferase activity upon co-transfection with miR-1203 (Figure3E).
SOCS3



ZNF853

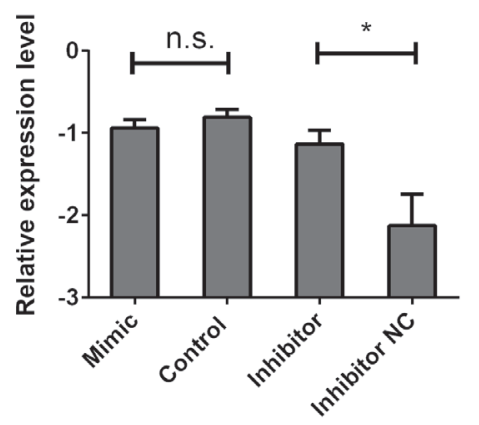

SOX12

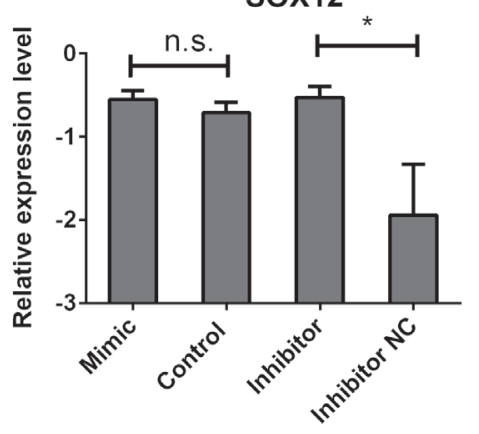

TRIM35

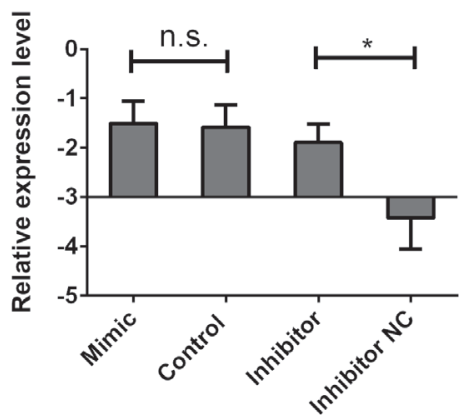

NKX3-2

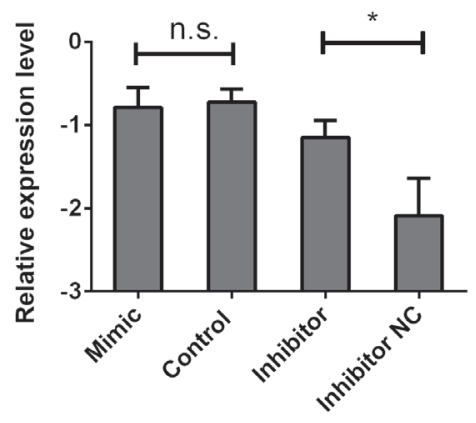

FOXK1

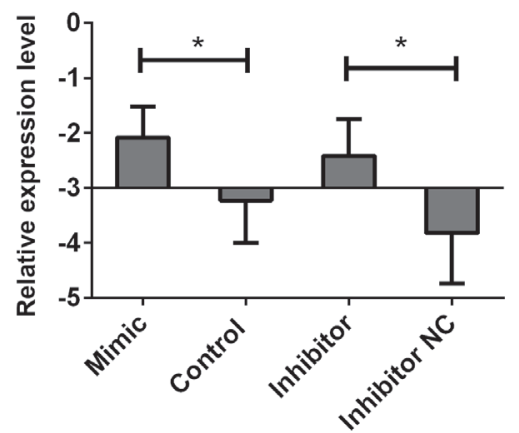

Figure 2. Relative expression of candidate genes of miR-1203. Relative expression of target genes of cells treated with miR-1203 mimics inhibitor. Data are presented as mean \pm SEM. ${ }^{*}$ indicates $\mathrm{p}<0.05,{ }^{*}$ indicates $\mathbf{p}<0.01$, n.s. indicated no significant. 
A

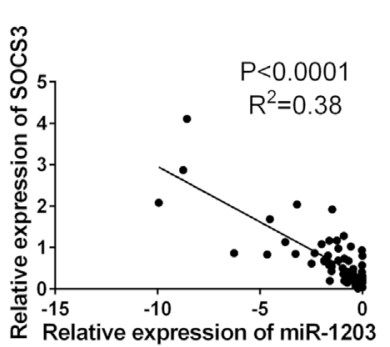

B

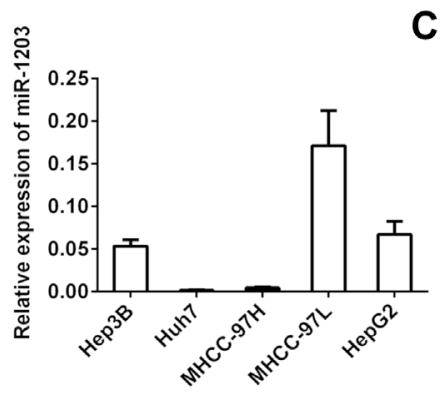

C

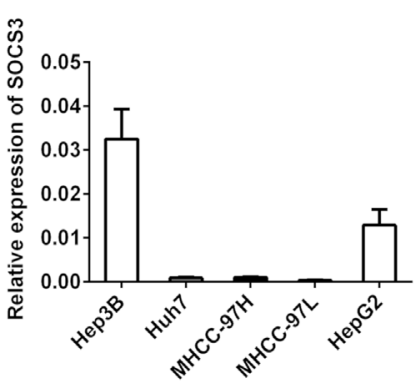

D

E

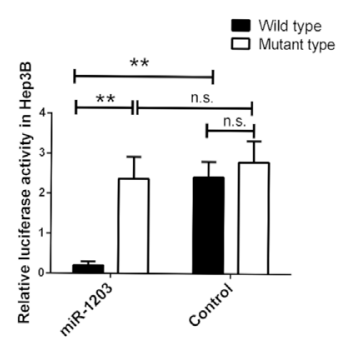

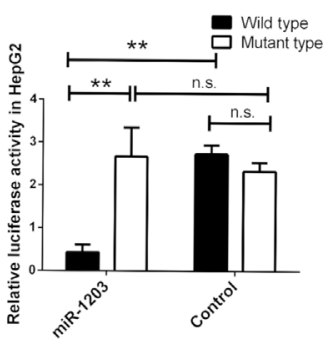

SOCS3 Wild Type 3'... CUGAGUCUCCAGCUUCUCCGGAG. . .5 miR-1203

CUCGACGUAGGACCGAGGCCC

SOCS3 Mutant 3'... CUGAGUCUCCAGCUUGAGGCCAG. . .5'

$F$

(

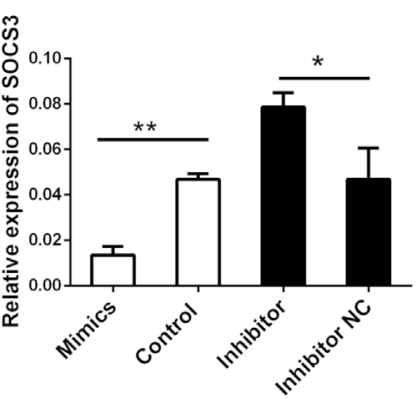

G

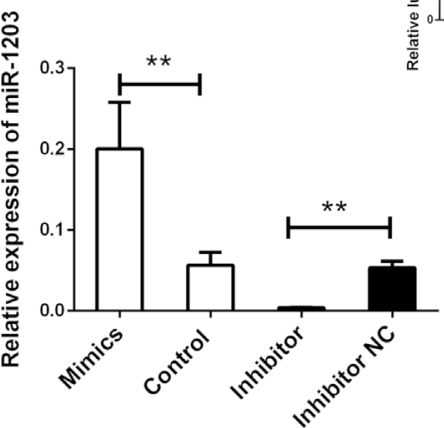

Inhibitor

Inhibitor Mimics Control control

SOCS3



GAPDH

Figure 3. miR-1203 decreased the expression of SOCS3 by directly binding to the 3'UTR. A) Pearson correction analysis of miR-1203 and SOCS3. B) Relative expression of miR-1203 in HCC cell lines. C) Relative expression of SOCS3 in HCC cell lines. D) Bioinformatics prediction of the binding site between the miR-1203 and SOCS3. The mutation types were also conducted into the pGL3 plasmid, as presented. E) HepG2 and Hep3B cells were co-transfected with miR-1203 mimics or control, Renilla luciferase vector pRL-SV40 and the full length of SOCS3 3'UTR or mutant 3'UTR. Both firefly and Renilla luciferase activities were measured in the same sample. Firefly luciferase signals were normalized with Renilla luciferase signals. Cells treated with the miRNA control were normalized to $100 \%$. F) Relative expression of SOCS3 mRNA and miR-1203 after treated with mimics or inhibitor. G) SOCS3 protein expression level. All tests were performed in triplicate and presented as mean \pm SEM. ${ }^{\star}$ indicates $p<0.05$, while ${ }^{* *}$ indicates $\mathbf{p}<0.01$

The effect of miR-1203 on SOCS3 mRNA levels was examined by quantifying the mRNA levels of SOCS3, while the protein level was measured by western blot. We found that the expression of SOCS3 was suppressed by the cells treated with miR-1203 mimics, which was increased by inhibiting miR-1203 (Figures 3F and 3G).

MiR-1203 promotes cell invasion in vitro. Clinicopathologic relevance analysis indicated that miR-1203 participates in HCC metastases. To confirm this finding, we performed a Transwell assay to detect the functional role of miR-1203 in cell invasion in both Hep3B and HepG2 cell lines. The
Table 2. Bioinformatics prediction of potential target genes.

\begin{tabular}{llllll}
\hline microRNA & $\begin{array}{l}\text { Related } \\
\text { Gene }\end{array}$ & miRbase & $\begin{array}{l}\text { Target } \\
\text { Scan }\end{array}$ & PicTar & $\begin{array}{l}\text { miRNA } \\
\text { Target }\end{array}$ \\
\hline hsa-miR-1203 & SOCS3 & $\sqrt{ }$ & $\sqrt{ }$ & $\sqrt{ }$ & $\sqrt{ }$ \\
& SOX12 & $\sqrt{ }$ & $\sqrt{ }$ & $\sqrt{ }$ & $\sqrt{ }$ \\
& NKX3-2 & & $\sqrt{ }$ & $\sqrt{ }$ & $\sqrt{ }$ \\
& ZNF853 & $\sqrt{ }$ & $\sqrt{ }$ & $\sqrt{ }$ \\
& TRIM35 & $\sqrt{ }$ & $\sqrt{ }$ & $\sqrt{ }$ & \\
& FOXK1 & $\sqrt{ }$ & $\sqrt{ }$ & $\sqrt{ }$ & \\
\hline
\end{tabular}


migrated cells were significantly increased in Hep3B treated with miR-1203 mimics, while this effect could be attenuated by the supplement of SOCS3. Reversely, the invasiveness was reduced by suppressing the expression of miR-1203, while this effect could be induced by the loss of SOCS3 (Figure 4A). The number of migrated cells is shown in Figure 4B. Based on the results above, we proposed that miR-1203 promoted cell invasion in HCC. The same results were obtained in Hep3B cell line (Figures 4C and 4D). Next we also measure the function of miR-1203 on cell proliferation and apoptosis by CCK and flow cytometry, respectively, and no signifi- cant effect was obtained (Figures 5A and 5B), indicating the miR-1203 might promote cell invasion during the development of human HCC.

MiR-1203 and SOCS3 acting as the fingerprint by predicting poor prognosis of HCC. It has been previously reported that miRNA could act as a biomarker for predicating the progression or prognosis of disease. Because we were curious about the translation of miR-1203 in clinical life, we attempted to detect the pattern of miR-1203 expression in plasma samples of patients used in microarrays. We found that circulating miR-1203 expression was aberrantly
A
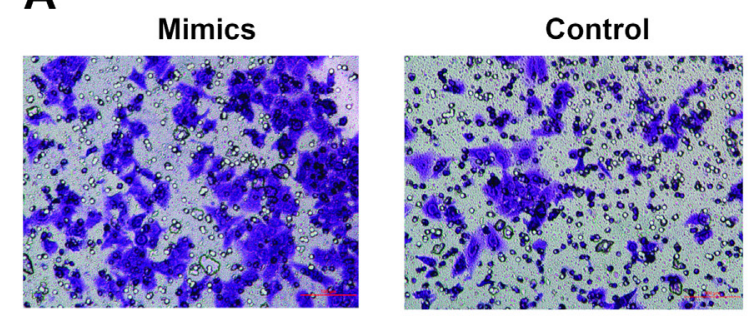

Inhibitor

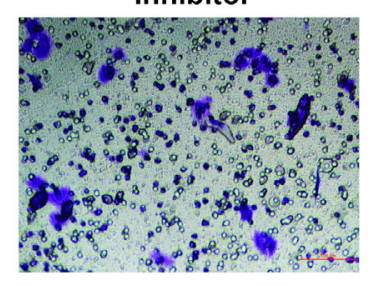

C

Mimics

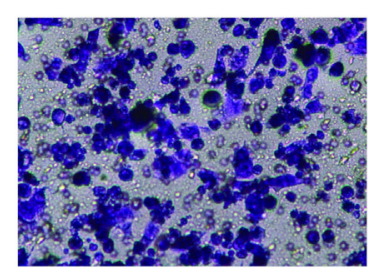

Inhibitor

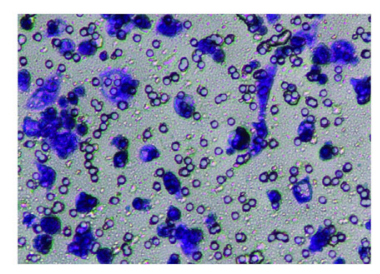

Inhibitor NC

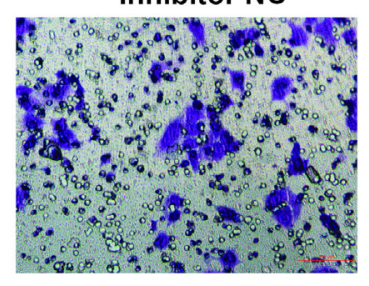

Control



Inhibitor NC

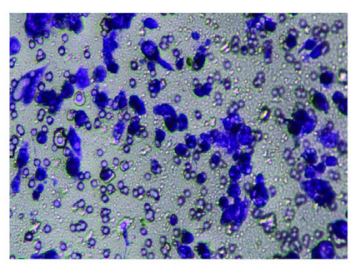

Mimics + SOCS3 OV
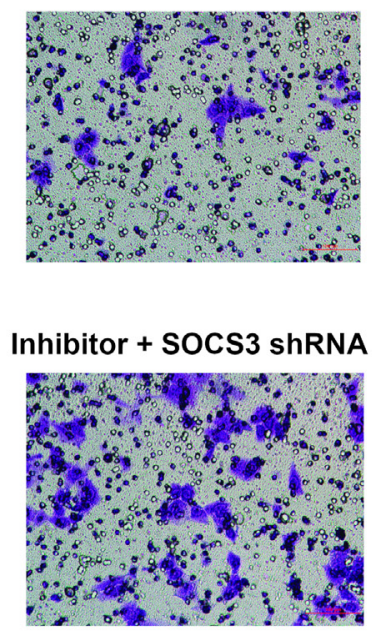

Mimics + SOCs3 OV



Inhibitor + SOCS3 shRNA

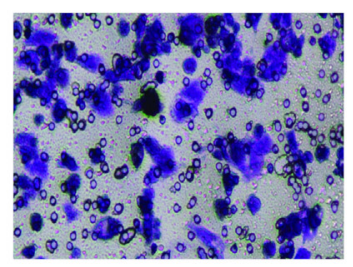

B

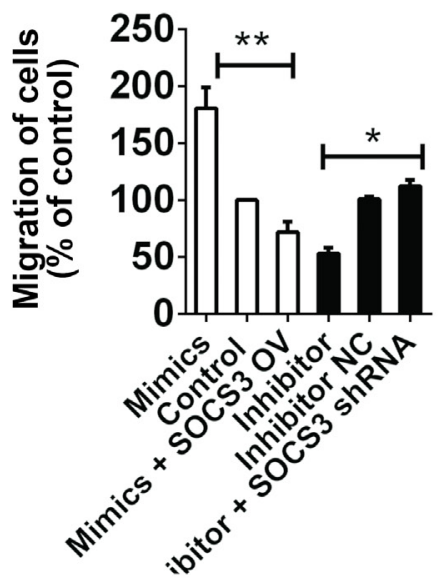

D

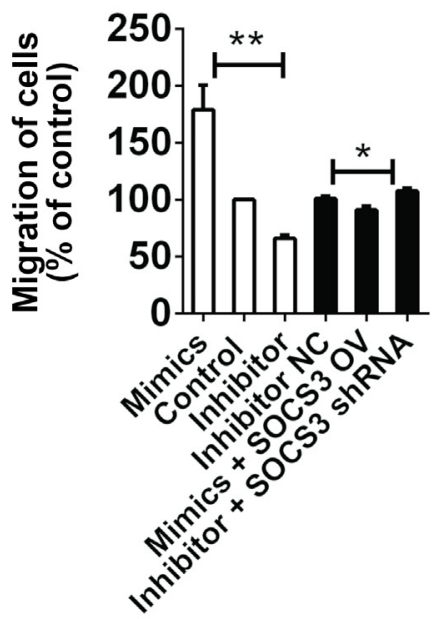

Figure 4. miR-1203 promoted cell invasion. A) Cell morphology graph of invasive cells in Hep3B cells after transfection of miR-1203 mimics, inhibitor, mimics plus SOCS3 overexpression lentivirus or inhibitor plus SOCS3 shRNA lentivirus. Magnification: 200x. B) Number of cells treated with control plasmid was normalized to $100 \%$; data are presented by means \pm SEM based on at least three independent experiments. C) Cell morphology graph of invasive cells in HepG2 cells after transfection of miR-1203 mimics, inhibitor, mimics plus SOCS3 overexpression lentivirus or inhibitor plus SOCS3 shRNA lentivirus. Magnification: $200 \times,{ }^{\star}$ indicates $\mathrm{p}<0.05$, while ${ }^{\star *}$ indicates $\mathrm{p}<0.01$. 
A

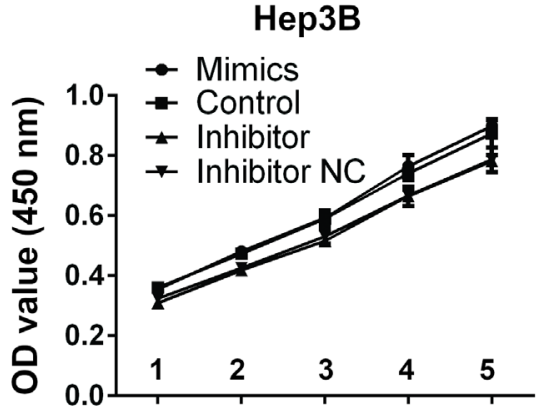

Time (day)

B

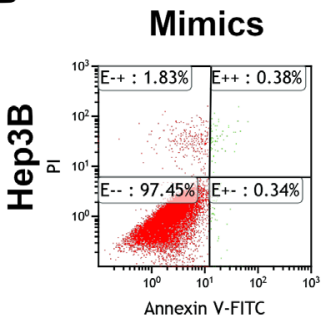

Mimics



Control

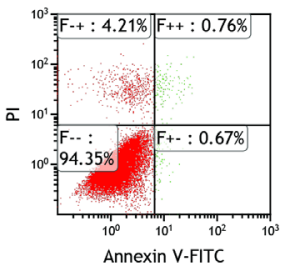

Control

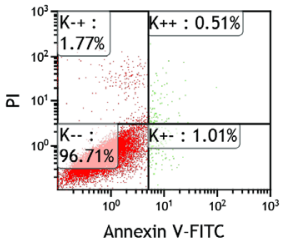

HepG2

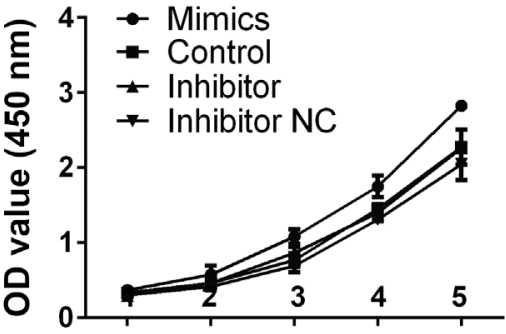

Time (day)

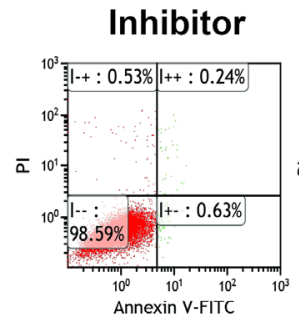

Inhibitor NC

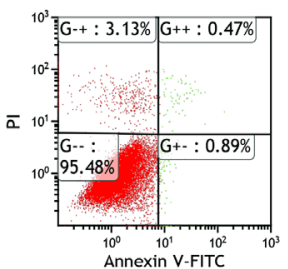

Inhibitor NC
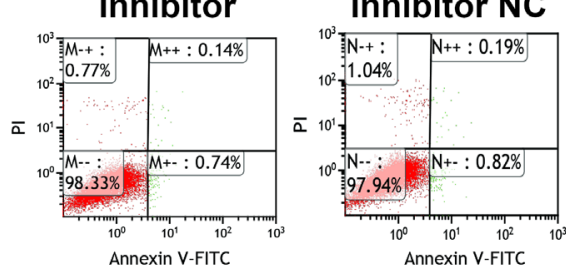

Figure 5. miR-1203 presented no regulation on cell proliferation or apoptosis. A) The cell proliferation was measured by CCK8 after treated with 1-5 days. B) The apoptosis was detected by flow cytometry. Data are presented by means \pm SEM based on at least three independent experiments.

increased in patients with metastases compared with patients without metastases or control patients (Figure 6A). Next, we analyzed whether circulating miR-1203 predicted HCC metastasis based on receiver operating characteristic (ROC) curves. The non-metastasis group was regarded as the control and the area under the curve was 0.67 (Figure 6B).

Previous reports showed that miRNA acts as a biomarker for predicting tumor growth. Kaplan-Meier analysis was used to determine whether miR-1203 expression is associated with overall survival (OS) and relapse-free survival (RFS) of HCC patients. HCC samples were divided into high $(n=40)$ and low $(n=40)$ expression groups, and the median was used as the cut-off value. The lower the RFS, the higher the expression of miR-1203 was observed (Figure 6C). The difference between the high and low expression groups was statistically significant $(\mathrm{p}=0.002)$. Similarly, a statistically significant association between miR-1203 with OS was also demonstrated ( $p=0.003$; Figure 6D). Besides, the RFS and OS for SOCS3 was also calculated, as presented in Figures $6 \mathrm{E}$ and $6 \mathrm{~F}$, lower expression of SOCS3 indicated poor RFS $(\mathrm{p}=0.001)$ and $\mathrm{OS}(\mathrm{p}=0.002)$. Furthermore, Cox proportional regression analysis indicated that the levels of miR-1203 and SOCS3 expression were independent prognostic factors for HCC patients (adjusted hazard ratio $=3.122,95 \%$ confidence interval $=2.113-5.121$, and $\mathrm{p}$-value $=0.026$; adjusted hazard ratio $=1.442,95 \%$ confidence interval $=1.992-8.901$, and $\mathrm{p}$-value $=0.011$, respectively, Table 3 ).

\section{Discussion}

Dysregulation of miRNAs and target genes are linked to tumor initiation and progression $[22,23]$. Thus far, miR-1203 has been identified as a potential biomarker for the diagnosis of prostate cancer in $75 \%$ of disseminated PC patients when compared to localized PC [24]. In addition, miR-1203 predicts post-operative prognosis, especially for tumor relapse in patients with small cell carcinoma [25]. Methylated miR-1203 has also been found to be associated with childhood obesity [26]; however, the detailed mechanism underlying the miR-1203 effect is poorly understood. In the current 


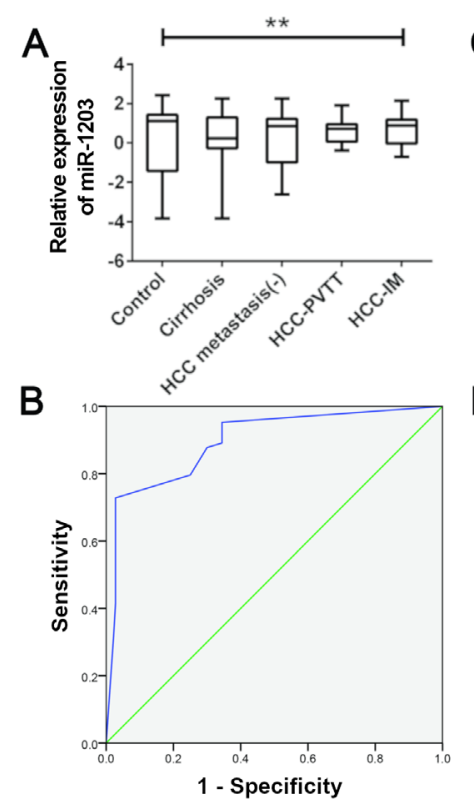

\section{C}
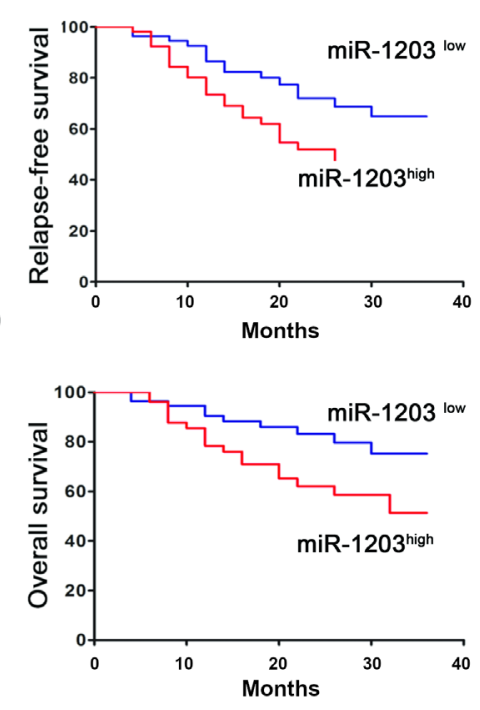

E
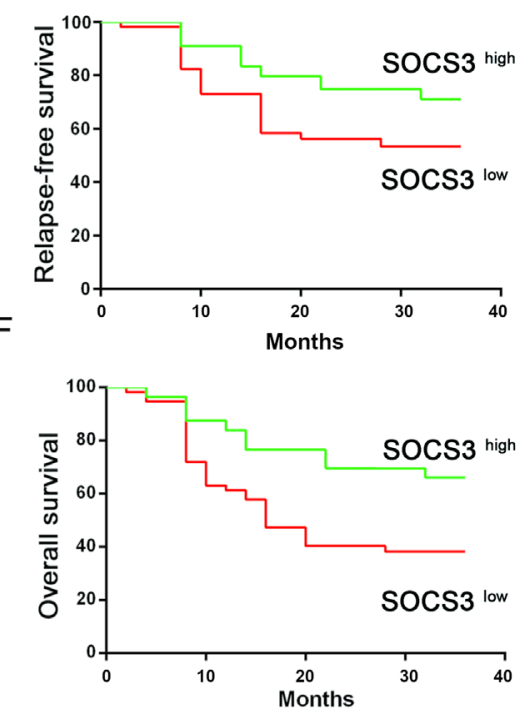

Table 3. Cox proportional hazards analysis (multivariable analysis).

\begin{tabular}{lccc}
\hline \multirow{2}{*}{ Clinical factor } & \multirow{2}{*}{ p-value } & \multicolumn{2}{c}{ Multivariable analysis } \\
\cline { 3 - 4 } & & aHR & 95\%CI \\
\hline miR-1203 & 0.026 & 3.122 & $2.113-5.121$ \\
SOCS3 & 0.011 & 1.442 & $1.992-8.901$ \\
Age & 0.142 & 1.222 & $0.992-1.242$ \\
Gender & 0.881 & 0.867 & $0.333-1.986$ \\
Cirrhosis & 0.251 & 1.494 & $0.921-1.888$ \\
ALT & 0.331 & 1.012 & $0.532-2.342$ \\
AFP & 0.424 & 0.911 & $0.712-3.229$ \\
Grade & 0.012 & 3.442 & $1.588-8.772$ \\
\hline
\end{tabular}

* indicates p<0.05; HR: Hazard ratio, AHR: Adjusted hazard ratio, CI: Confidence interval

study, we conducted experiments to demonstrate the pattern regulating metastasis-associated miR-1203 with SOCS3. The novel miR-1203 confirmed in our study is the first report in human HCC. Increased miR-1203 might inversely regulate SOCS3, resulting in promotion of cell invasion in vitro. Circulating miR-1203 was also detectable and might serve as a predictor of prognosis for HCC. Circulating miRNA has been approved for transport by vesicles or exosomes $[27,28]$. Tang et al. [26] has confirmed that circulating non-coding RNA expression is decreased after tumorectomy.

The suppressor of cytokine signaling (SOCS) gene family has been shown to play an essential role in suppressing tumor progression by inhibiting JAK/STAT and NFKB signaling, and promoting p53 signaling $[29,30]$. Among the
SOCS family, SOCS3 has been reported to be associated with various types of cancers [31]. For example, loss of SOCS3 in HCC is associated with STAT3 over-phosphorylation and poor prognosis [32]. It has been shown that inhibition of SOCS3 expression promotes STAT3 activation, enhances hepatic fibrosis, and increases proliferation and tumor aggressiveness [33]. MiR-19a/b and miR-203 negatively regulate SOCS3 in human cancers, which causes a decreased level of SOCS3 [34-36].

In conclusion, we identified a novel miR-1203 in human HCC, which is upregulated and highly associated with tumor metastases. The elevated level of miR-1203 expression decreased the level of SOCS3 via direct binding at 3'UTR, resulting in the promotion of cell invasion in vitro. We also showed that plasma miR-1203 significantly predicts tumor metastasis and a poor prognosis in patients with HCC. We propose that miR-1203 might be a novel index for clinical prognosis.

Acknowledgements: This study was financed by University-Level Science and Technology Project of Binzhou Medical University (BY2018KJ09).

\section{References}

[1] WU D, LIU G, LIU Y, SAIYIN H, WANG C et al. Zinc finger protein 191 inhibits hepatocellular carcinoma metastasis via discs large 1-mediated yes associated protein inactivation. Hepatology 2016; 64: 1148-1162. https://doi.org/10.1002/ hep. 28708 
[2] LIU Y, CHAI Y, ZHANG J, TANG J. A Function Variant at miR-501 Alters Susceptibility to Hepatocellular Carcinoma in a Chinese Han Population. Cell Physiol Biochem 2016; 38 : 2500-2508. https://doi.org/10.1159/000445600

[3] DE BARI B, OZSAHIN M, BIZE P, BOUSSAHA T, DEPLANQUE G et al. Can Stereotactic Body Radiotherapy Really Be Considered the Preferred Treatment in Large Hepatocellular Carcinoma? J Clin Oncol 2016; 34: 2798-2799. https://doi.org/10.1200/JCO.2016.66.7196

[4] DAUCH D, RUDALSKA R, COSSA G, NAULT JC, KANG TW et al. A MYC-aurora kinase A protein complex represents an actionable drug target in p53-altered liver cancer. Nat Med 2016; 22: 744-753. https://doi.org/10.1038/ nm.4107

[5] YUAN JH, YANG F, WANG F, MA JZ, GUO YJ et al. A long noncoding RNA activated by TGF-beta promotes the invasion-metastasis cascade in hepatocellular carcinoma. Cancer Cell 2014; 25: 666-681. https://doi.org/10.1016/j. ccr.2014.03.010

[6] MATHIAS RA, GOPAL SK, SIMPSON RJ. Contribution of cells undergoing epithelial-mesenchymal transition to the tumour microenvironment. J Proteomics 2013; 78: 545-557. https://doi.org/10.1016/j.jprot.2012.10.016

[7] LIU S, LI N, YU X, XIAO X, CHENG K et al. Expression of intercellular adhesion molecule 1 by hepatocellular carcinoma stem cells and circulating tumor cells. Gastroenterology 2013; 144: 1031-1041. https://doi.org/10.1053/j.gastro.2013.01.046

[8] TANG J, ZHUO H, ZHANG X, JIANG R, JI J et al. A novel biomarker Linc00974 interacting with KRT19 promotes proliferation and metastasis in hepatocellular carcinoma. Cell Death Dis 2014; 5: e1549. https://doi.org/10.1038/cddis. 2014.518

[9] ZHOU Q, ZHENG X, CHEN L, XU B, YANG X et al. Smad2/3/4 Pathway Contributes to TGF-beta-Induced MiRNA-181b Expression to Promote Gastric Cancer Metastasis by Targeting Timp3. Cell Physiol Biochem 2016; 39: 453466. https://doi.org/10.1159/000445638

[10] ROTHSCHILD SI, GAUTSCHI O, BATLINER J, GUGGER M, FEY MF et al. MicroRNA-106a targets autophagy and enhances sensitivity of lung cancer cells to Src inhibitors. Lung Cancer 2017; 107: 73-83. Https://doi.org/10.1016/j.lungcan.2016.06.004

[11] TO KK, LEUNG WW, NG SS. A novel miR-203-DNMT3bABCG2 regulatory pathway predisposing colorectal cancer development. Mol Carcinog 2017; 56: 464-477. https://doi. org/10.1002/mc.22508

[12] ZHU HT, HASAN AM, LIU RB, ZHANG ZC, ZHANG X et al. Serum microRNA profiles as prognostic biomarkers for HBV-positive hepatocellular carcinoma. Oncotarget 2016; 7: 45637-45648. https://doi.org/10.18632/oncotarget.10082

[13] CHEN Y, DU M, WANG J, XING P, ZHANG Y et al. MiRNA-200a expression is inverse correlation with hepatocyte growth factor expression in stromal fibroblasts and its high expression predicts a good prognosis in patients with nonsmall cell lung cancer. Oncotarget 2016; 7: 48432-48442. https://doi.org/10.18632/oncotarget.10302
[14] ObERG K, MODLIN IM, DE HERdER W, PAVEL M, KLIMSTRA D et al. Consensus on biomarkers for neuroendocrine tumour disease. Lancet Oncol 2015; 16: e435-e446. https://doi.org/10.1016/S1470-2045(15)00186-2

[15] CHITKARA D, MITTAL A, MAHATO RI. miRNAs in pancreatic cancer: therapeutic potential, delivery challenges and strategies. Adv Drug Deliv Rev 2015; 81: 34-52. https://doi. org/10.1016/j.addr.2014.09.006

[16] DVINGE H, GIT A, GRAF S, SALMON-DIVON M, CURTIS $\mathrm{C}$ et al. The shaping and functional consequences of the microRNA landscape in breast cancer. Nature 2013; 497: 378-382. https://doi.org/10.1038/nature12108

[17] CHEN JS, LIANG LL, XU HX, CHEN F, SHEN SL et al. miR-338-3p inhibits epithelial-mesenchymal transition and metastasis in hepatocellular carcinoma cells. Oncotarget 2016; 8: 71418-71429. https://doi.org/10.18632/oncotarget.10138

[18] NIWA Y, KANDA H, SHIKAUCHI Y, SAIURA A, MATSUBARA K et al. Methylation silencing of SOCS-3 promotes cell growth and migration by enhancing JAK/STAT and FAK signalings in human hepatocellular carcinoma. Oncogene 2005; 24: 6406-6417. https://doi.org/10.1038/sj.onc.1208788

[19] WU WY, KIM H, ZHANG CL, MENG XL, WU ZS. Loss of suppressors of cytokine signaling 3 promotes aggressiveness in hepatocellular carcinoma. J Invest Surg 2014; 27: 197-204. https://doi.org/10.3109/08941939.2013.873098

[20] XU G, YANG F, DING CL, WANG J, ZHAO P et al. MiR221 accentuates IFN's anti-HCV effect by downregulating SOCS1 and SOCS3. Virology 2014; 462-463: 343-350. https://doi.org/10.1016/j.virol.2014.06.024

[21] QUETIER I, BREZILLON N, DURIEZ M, MASSINET $\mathrm{H}$, GIANG E et al. Hepatitis B virus HBx protein impairs liver regeneration through enhanced expression of IL-6 in transgenic mice. J Hepatol 2013; 59: 285-291. https://doi. org/10.1016/j.jhep.2013.03.021

[22] BLOOMSTON M, FRANKEL WL, PETROCCA F, VOLINIA S, ALDER H et al. MicroRNA expression patterns to differentiate pancreatic adenocarcinoma from normal pancreas and chronic pancreatitis. JAMA 2007; 297: 1901-1908. https://doi.org/10.1001/jama.297.17.1901

[23] HU Z, CHEN X, ZHAO Y, TIAN T, JIN G et al. Serum microRNA signatures identified in a genome-wide serum microRNA expression profiling predict survival of non-smallcell lung cancer. J Clin Oncol 2010; 28: 1721-1726. https:// doi.org/10.1200/JCO.2009.24.9342

[24] HALDRUP C, KOSAKA N, OCHIYA T, BORRE M, HOYER $S$ et al. Profiling of circulating microRNAs for prostate cancer biomarker discovery. Drug Deliv Transl Res 2014; 4: 19-30. https://doi.org/10.1007/s13346-013-0169-4

[25] OKUMURA T, SHIMADA Y, OMURA T, HIRANO K, NAGATA T et al. MicroRNA profiles to predict postoperative prognosis in patients with small cell carcinoma of the esophagus. Anticancer Res 2015; 35: 719-727.

[26] MANSEGO ML, GARCIA-LACARTE M, MILAGRO FI, MARTI A, MARTINEZ JA et al. DNA methylation of miRNA coding sequences putatively associated with childhood obesity. Pediatr Obes 2017; 2: 19-27. https://doi.org/10.1111/ ijpo.12101 
[27] IAVELLO A, FRECH VS, GAI C, DEREGIBUS MC, QUESENBERRY PJ et al. Role of Alix in miRNA packaging during extracellular vesicle biogenesis. Int J Mol Med 2016; 37: 958-966. https://doi.org/10.3892/ijmm.2016.2488

[28] KOBERLE V, KAKOSCHKY B, IBRAHIM AA, SCHMITHALS C, PEVELING-OBERHAG J et al. Vesicleassociated microRNAs are released from blood cells on incubation of blood samples. Transl Res 2016; 169: 40-46. https:// doi.org/10.1016/j.trsl.2015.10.010

[29] STAHL A, JOYAL JS, CHEN J, SAPIEHA P, JUAN AM et al. SOCS3 is an endogenous inhibitor of pathologic angiogenesis. Blood 2012; 120: 2925-2929. https://doi.org/10.1182/ blood-2012-04-422527

[30] LI Y, DE HAAR C, CHEN M, DEURING J, GERRITS MM et al. Disease-related expression of the IL6/STAT3/SOCS3 signalling pathway in ulcerative colitis and ulcerative colitisrelated carcinogenesis. Gut 2010; 59: 227-235. https://doi. org/10.1136/gut.2009.184176

[31] PAUKKU K, SILVENNOINEN O. STATs as critical mediators of signal transduction and transcription: lessons learned from STAT5. Cytokine Growth Factor Rev 2004; 15: 435455. https://doi.org/10.1016/j.cytogfr.2004.09.001
[32] Geng L, Lu K, Li P, Li X, Zhou X, Li Y, Wang X: GLI1 inhibitor GANT61 exhibits antitumor efficacy in T-cell lymphoma cells through down-regulation of p-STAT3 and SOCS3. Oncotarget 2017; 8: 48701-48710. https://doi.org/10.18632/oncotarget. 9792

[33] JIANG BG, YANG Y, LIU H, GU FM, YANG Y et al. SOCS3 Genetic Polymorphism Is Associated With Clinical Features and Prognosis of Hepatocellular Carcinoma Patients Receiving Hepatectomy. Medicine (Baltimore) 2015; 94: e1344. https://doi.org/10.1097/MD.0000000000001344

[34] LI HW, XIE Y, LI F, SUN GC, CHEN Z et al. Effect of miR19a and miR-21 on the JAK/STAT signaling pathway in the peripheral blood mononuclear cells of patients with systemic juvenile idiopathic arthritis. Exp Ther Med 2016; 11: 25312536. https://doi.org/10.3892/etm.2016.3188

[35] NAVARRO A, PAIRET S, ALVAREZ-LARRAN A, PONS A, FERRER G et al. miR-203 and miR-221 regulate SOCS1 and SOCS3 in essential thrombocythemia. Blood Cancer J 2016; 6: e406. https://doi.org/10.1038/bcj.2016.10

[36] CHENG X, ZHANG X, SU J, ZHANG Y, ZHOU W et al. miR-19b downregulates intestinal SOCS3 to reduce intestinal inflammation in Crohn's disease. Sci Rep 2015; 5: 10397. https://doi.org/10.1038/srep10397 